\title{
Surface Morphologies of He-Implanted Tungsten
}

\author{
M. E. Bannister ${ }^{1,}{ }^{,}$, F. W. Meyer ${ }^{1}$, H. Hijazi ${ }^{1,9}$, K. A. Unocic ${ }^{2}$, L. M. Garrison ${ }^{2}$, and C. M. Parish ${ }^{2}$ \\ ${ }^{1}$ Physics Division, Oak Ridge National Laboratory, Oak Ridge, TN 37831-6371 USA \\ ${ }^{2}$ Materials Science and Technology Division, Oak Ridge National Laboratory, Oak Ridge, TN, USA
}

\begin{abstract}
Surface morphologies of tungsten surfaces, both polycrystalline and single-crystal [110], were investigated using SEM and FIB/SEM techniques after implantations at elevated surfaces temperatures (1200-1300 K) using well-characterized, mono-energetic He ion beams with a wide range of ion energies (218 eV to $250 \mathrm{keV}$ ). Nanofuzz was observed on polycrystalline tungsten (PCW) following implantation of 100-keV He ions at a flux threshold of $0.9 \times 10^{16} \mathrm{~cm}^{-2} \mathrm{~s}^{-1}$, but not following 200-keV implantations with similar fluxes. No nanofuzz formation was observed on single-crystal [110] tungsten (SCW), despite fluxes exceeding those demonstrated previously to produce nanofuzz on polycrystalline tungsten. Predamaging the single-crystal tungsten with implanted $\mathrm{C}$ impurity interstitials did not significantly affect the surface morphologies resulting from the high-flux He ion implantations. The main factor leading to the different observed surface structures for the pristine and C-implanted single-crystal W samples appeared to be the peak He ion flux characterizing the different exposures. It was speculated that nanofuzz formation was not observed for any SCW target exposures because of increased incubation fluences required for such targets.
\end{abstract}

Keywords: He nanofuzz formation, tungsten surface modification, plasma wall interactions

\footnotetext{
*Corresponding author: bannisterme@ornl.gov

"Present address: Aix-Marseille Université, CNRS, PIIM UMR 7345, F-13397 Marseille, France
}

This manuscript has been authored by UT-Battelle, LLC under Contract No. DE-AC05-00OR22725 with the U.S. Department of Energy. The United States Government retains and the publisher, by accepting the article for publication, acknowledges that the United States Government retains a non-exclusive, paidup, irrevocable, world-wide license to publish or reproduce the published form of this manuscript, or allow others to do so, for United States Government purposes. The Department of Energy will provide public access to these results of federally sponsored research in accordance with the DOE Public Access Plan (http://energy.gov/downloads/doe-public-access-plan). 


\section{Introduction}

Tungsten is the leading candidate for plasma-facing material in next-generation magnetic fusion devices due to possessing properties needed in such an extreme environment, namely a high melting point, high thermal conductivity, and a combination of high sputtering energy threshold and low sputter yield.

Recent experiments [Takamura 2006, Baldwin 2008] on linear plasma devices studying the interaction of hot tungsten surfaces with He ions, the byproducts of D-T fusion reactions, discovered resultant morphological changes, even at relatively low ion impact energies on the order of $100 \mathrm{eV}$, with the production of nanofuzz, nanometer scale tendrils of tungsten growing on the surface. The detailed sequence of fundamental processes leading to nanofuzz growth is still actively debated, and a number of alternate scenarios have been proposed [Kajita 2009, Krasheninnikov 2011, Sefta 2013, Lasa 2014]. Although most of the research to date has focused on nanofuzz formation on polycrystalline tungsten, several recent studies \{Baldwin 2010, Kajita 2014] have also investigated nanofuzz growth on singlecrystal tungsten targets.

Nanofuzz is very undesirable in fusion devices as it degrades the very properties making tungsten the prime plasma-facing material - reducing the thermal conductivity, and yielding a potential source of deleterious high- $Z$ dust impurity. A fundamental science understanding is needed to provide a rational basis for development of fuzz-resistant materials.

The present paper reports investigations into the underlying mechanisms leading to nanofuzz growth by He-ion-implantation in hot tungsten surfaces. For He-ion impact energies above the displacement damage threshold, trapping sites are available at radiation-induced vacancies and interstitials, though the role of such sites needs much elucidation. To investigate the influence of damage induced by the implanted He ions, previous investigations of nanofuzz growth on polycrystalline tungsten (PCW) for impact energies of $12 \mathrm{keV}$ and less [Meyer 2014, Meyer 2016b] were extended to energies up to 200 $\mathrm{keV}$. Surface morphologies were studied with scanning electron microscopy (SEM) and transmission electron microscopy (TEM), including imaging of the near-surface cross sections by employing a Focused Ion Beam (FIB) tool. Thermal desorption spectroscopy (TDS) was employed following high-energy exposures to gain further insight into the role of ion-induced damage during the early stages of nanofuzz production.

For He-ion impact energies below the displacement damage threshold, the growth of nano-structures must be due to He trapping at intrinsic or extrinsic defect sites, followed by the dynamics of nearsurface cluster nucleation, bubble formation, coalescence, growth, and ultimately bursting [Sefta 2013]. The exact mechanisms and processes involved are still not understood. At all energies, the effects of extrinsic trapping sites due to impurity interstitials have not been clearly established. In order to gain insights into the fundamental mechanisms leading to nanofuzz growth, experiments were performed under well-characterized and well-defined conditions for pristine W single-crystal targets free of extrinsic impurity trap sites to isolate the He-W interactions in a defect-free environment. 


\section{Experiment}

The present experiments were carried out at the Multicharged-Ion Research Facility (MIRF) at Oak Ridge National Laboratory. This facility is uniquely capable of investigating He-ion induced nanofuzz growth on hot tungsten under well-controlled conditions. Two electron-cyclotron-resonance (ECR) ion sources produce high-flux, mono-energetic ion beams with energies tunable from about $100 \mathrm{eV}$ to $250 \mathrm{keV}$. The He ion beams, in turn, are well-characterized using advanced diagnostics, beam imaging and fluxmapping techniques to maximize ion flux while accurately quantifying exposures. An ultra-high vacuum environment maintains pristine, well-characterized surfaces. The ECR ion sources also allow the production of ion-beam-induced radiation damage and introduction of interstitial impurity ions through implantation.

The polycrystalline tungsten (PCW) targets, measuring $12.7 \times 12.7 \times 0.64 \mathrm{~mm}$, were cut from rolled sheet stock and mechanically polished to a mirror-like finish [Meyer 2014]. Earlier x-ray photoelectron spectroscopy (XPS) analysis [Meyer 2016b] of these targets showed up to 5\% C impurities to depths up to $100 \mathrm{~nm}$. Subsequent analysis by Luvak Laboratories [www.luvak.com] using accepted bulk analytical techniques found our tungsten sheet stock to be greater than $99.97 \%$ pure, with Cu the main impurity at $0.022 \%$. Bulk C impurity was detected only at the $0.002 \%$ level. Similar differences in surface and bulk C impurity levels were observed for PLANSEE W (99.9999\% purity) at PISCES [Baldwin 2008] following plasma exposures, and are most likely from $C$ sources in the vacuum and/or plasma environment that the $W$ targets are exposed to. The single-crystal tungsten (SCW) samples had a [110] surface orientation and were elliptical in shape, with an approximately $7 \mathrm{~mm}$ size and $0.4 \mathrm{~mm}$ thickness. The SCW samples were mechanically polished and subsequently electro-polished in an aqueous solution of potassium hydroxide.

For ion energies of $12 \mathrm{keV}$ and below, a Caprice ECR ion source [Meyer 2000] is used. A high-flux deceleration module [Hijazi 2013] positioned upstream of the tungsten targets permits implantation energies down to $100 \mathrm{eV}$ or less; a beam flux monitor [Bannister 2014] is used to optimize the decel optics to maximize and quantify the ion flux as a function of position on the target. All the implantations reported in this paper were performed at normal incidence, although some experiments have been performed at other angles [Meyer 2016a]. The target is positioned in the ion beam with a three-axis manipulator and features electron-beam sample heating up to $1500 \mathrm{~K}$. The temperature of the tungsten target during exposure is measured with a type $\mathrm{K}$ thermocouple. In addition, the emissivity of the target is monitored during exposure with a digital camera, permitting observation of nanofuzz formation in real-time, since the emissivity of the nanofuzz differs significantly from that of unexposed tungsten. This technique was employed in a systematic study [Meyer 2016b] of the incubation period [Baldwin 2009, Kajita 2011] of nanofuzz growth - the time required for an ion beam of sufficient flux to produce detectable fuzz. The real-time sample imaging also established that the nanofuzz growth has a flux threshold, as the size of the nanofuzz region on the tungsten targets did not grow with time (fluence) to fill the entire beam spot, but was observed only where the ion beam flux was above a critical value

For ion energies above $12 \mathrm{keV}$, an all-permanent-magnet ECR ion source installed on a 250-kV high voltage platform [Meyer 2006] is employed. As with the low-energy implantation setup, the tungsten 
targets are positioned in the ion beam using a three-axis manipulator with electron-beam heating capable of producing sample temperatures up to $1500 \mathrm{~K}$. The temperature of the tungsten targets during exposure are monitored with a type $\mathrm{N}$ thermocouple. The ion beam at the target position is optimized and characterized by a beam flux monitor with a $0.5 \mathrm{~mm}$ aperture. All implantation was performed with the ion beam normal to the tungsten surface.

In separate low-flux exposures in the high-energy beamline, tungsten targets were also studied with thermal desorption spectroscopy of the implanted He. The parameters of the electron beam sample heater were optimized to produce a linear time ramp of the target temperature while the released $\mathrm{He}$ atoms were measured with a residual gas analyzer (RGA). Free molecular flow of He from a reservoir of known pressure (less than 0.1 Torr) through a $0.057 \mathrm{~cm}$ inner diameter capillary was used as a calibrated leak to put the RGA measurements on an absolute scale. Thermal desorption spectra were measured several minutes after He ion exposures were complete, and with the implantation section of the vacuum beam line isolated from the ECR ion source by a gate valve to prevent He gas from the source increasing the background for the TDS measurements.

\section{Results and Discussion}

A previous study [Meyer 2016b] of low-energy implantation of He ions in PCW targets established flux thresholds of a few times $10^{16} \mathrm{~cm}^{-2} \mathrm{~s}^{-1}$ for nanofuzz formation as a function of ion energy in the range $218 \mathrm{eV}$ to $8.5 \mathrm{keV}$. A model was developed that reproduced the observed energy dependence of the flux thresholds in that energy range, taking into account ion implantation depth, He ion reflection, and trapping due to impurities and induced damage. To determine whether such dependence continues at much higher energies and to gain further insight into the importance of ion-induced damage on nanofuzz formation, He-ion implantation studies on PCW targets were extended to ion energies of 100$200 \mathrm{keV}$ in the present investigation. These experiments also sought to answer the question as to whether there is an upper energy limit for fuzz formation, since higher energy ions are deposited at and create damage at greater depths during implantation, while nanofuzz formation appears to be a nearsurface phenomenon.

Figure 1 shows SEM and FIB/SEM images of PCW targets implanted with He ions at $100 \mathrm{keV}$ and $200 \mathrm{keV}$ and, for comparison, $218 \mathrm{eV}$. Nanofuzz formation is clearly visible (Fig. 1-b) for the PCW implanted with a peak flux of $1.9 \times 10^{16} \mathrm{~cm}^{-2} \mathrm{~s}^{-1}$ ions at $100 \mathrm{keV}$ with a $\mathrm{W}$ temperature of $1200 \mathrm{~K}$, and $4300 \mathrm{sec}$ exposure time. The tendrils of the nanofuzz are coarser than those seen in Fig. 1-a for the $218 \mathrm{eV}$ implantation $\left(3.1 \times 10^{16} \mathrm{~cm}^{-2} \mathrm{~s}^{-1}\right.$ at $\left.1300 \mathrm{~K}, 1800 \mathrm{sec}\right)$, but the thickness of the fuzz layer is not significantly greater as seen in the FIB/SEM images (Figs. 1-d,e). From the dimensions of the nanofuzz spot for this exposure and the measured flux map, the threshold for fuzz formation at $100 \mathrm{keV}$ was determined to be $0.9 \times 10^{16}$ $\mathrm{cm}^{-2} \mathrm{~s}^{-1}$, consistent with the trend of decreasing flux thresholds at energies in the keV energy range found previously [Meyer 2016b]. The SEM image (Fig. 1-c) for the $200 \mathrm{keV}$ implantation (peak flux $1.0 \mathrm{x}$ $10^{16} \mathrm{~cm}^{-2} \mathrm{~s}^{-1}$ at $1250 \mathrm{~K}$, and $2700 \mathrm{sec}$ exposure time) shows pinholes created during implantation, but no nanofuzz is evident, despite a fluence equal to that for the $100-\mathrm{keV}$ implantation, suggesting that the bulk of the $\mathrm{He}$ is implanted and trapped too deeply below the surface to reach the critical near-surface He volume density rate of change required for fuzz formation. More detailed comparison with [Meyer 
2016b] is unfortunately not possible, since there was no real-time imaging of the sample during irradiation, so the incubation fluence for the high energy exposure could not be determined. No FIB/SEM imaging was performed for the $200-k e V$ implanted sample since no nanofuzz was observed.

To study the effects of ion-induced damage on He trapping in tungsten and to gain insight into the nucleation process that is a pre-cursor to nanofuzz formation, TDS measurements were performed on PCW targets implanted with lower flux $\left(4.3 \times 10^{15} \mathrm{~cm}^{-2} \mathrm{~s}^{-1}\right) 100 \mathrm{keV}$ ions, and at $575 \mathrm{~K}$, a temperature well below the minimum needed for nanofuzz formation [Kajita 2009]. Figure 2 shows helium thermal desorption spectra measured at a heating rate of $15 \mathrm{~K} / \mathrm{s}$ after each of three sequential implantations with average fluences of $0.5,1.0$, and $2.0 \times 10^{18} \mathrm{~cm}^{-2}$, with no annealing between the exposures. Using SRIM [Ziegler 1985] to calculate the ion-induced damage according to the method of Stoller [Stoller 2013], estimated accumulated mean damage levels in the PCW after each exposure were $4.7 \mathrm{dpa}, 14.1$ dpa, and 32.9 dpa, respectively. Numerically integrating the He desorption rates as a function of time during the TDS heating ramps and knowing the total number of He ions incident on the PCW target during each exposure, one can determine retention fractions for the $\mathrm{He}$ of $30.4 \%, 53.6 \%$, and $35.0 \%$, for the three successive exposures The drop in retention during the last implantation could be due to beam induced damage saturation reducing the associated He trapping [Fu 2004]. The measured high-energy retention fractions are significantly larger than those observed at energies below $1 \mathrm{keV}$, where retention fractions in the range of a few to 10 percent have been reported [Lee 2007] in the present fluence range, albeit at somewhat different target temperatures than our own. The peaks in the spectra near $850 \mathrm{~K}$ are likely due to intrinsic trapping sites with lower binding energies, whereas the larger peaks near $1350 \mathrm{~K}$ can be attributed to trapping sites associated with accumulating damage created by the implanted He ions [Kornelsen 1970, Kornelsen 1972]. The quadratic increase of the peak heights near $1350 \mathrm{~K}$ with fluence for the two lowest fluences --fluence doubles while the peak height increases by a factor of more than four-- is consistent with trapping at beam-induced damage sites [Kornelson 1970]. At the highest fluence, a breakdown in this quadratic scaling is seen, most likely due to saturation effects but possibly also due to pinhole formation, although no SEM imaging was performed for this exposure to test this possibility. The $1350 \mathrm{~K}$ peak temperature is consistent with formation of clusters containing between 15 and $20 \mathrm{He}$ atoms [Kornelsen 1980]. TDS measurements were not performed on the PCW targets exposed under conditions leading to nanofuzz growth prior to SEM and FIB/SEM imaging because the heating of the targets during the TDS could lead to annealing out much of the nanofuzz produced [Wright 2015, Meyer 2016b]. Future TDS measurements of these targets with observed nanofuzz are planned now that the SEM analyses are complete.

We also carried out measurements of He ion induced surface morphology changes in [110] single crystal $\mathrm{W}$ samples. These measurements were a follow up to previous measurements using polycrystalline samples which indicated significant dependence of the initial morphological surface changes induced by low energy $\mathrm{He}$ ion beams on the orientation of the examined surface grain [Parish 2013]. In addition, by eliminating or greatly reducing grain boundary effects, their effect on nanofuzz formation flux thresholds [Meyer 2016b] could be investigated. Table 1 summarizes the conditions under which four different exposures were carried out. All exposures were carried out at a nominal target temperature of $1000{ }^{\circ} \mathrm{C}$ where we had previously observed nanofuzz formation on polycrystalline $\mathrm{W}$ samples [Meyer 
2016b]. Even though the peak He ion fluxes used for the single crystal target exposures were significantly above the flux thresholds for nanofuzz formation found for our earlier polycrystalline targets, SEM imaging of the [110] W surfaces after He exposure showed no evidence of nanofuzz formation. Instead of nanofuzz, the following sequence of surface morphologies was found, as illustrated in Figure 3. At low fluxes and fluences, i.e. sufficiently far away from the beam peak intensity, irrespective of He ion energy, the surfaces showed pinholes and minor surface roughening (Fig. 3-a). With increasing beam flux, the formation of orderly ripples was observed (Fig. 3-b), whose ridges were preferentially aligned with the $<001>$ direction (see TEM image in Fig. 4-c, and electron diffraction pattern in Fig. 4-d). This preferred ridge alignment has been noted elsewhere [Miyamoto 2014; Garrison 2015; Ohno 2013; Kajita 2014] for a range of different energy and flux conditions. With further increasing flux, the ripples became less ordered with shorter unbroken ridge lengths and appeared progressively more tangled, eventually giving the appearance of well mixed spaghetti (Fig. 3-C). Compared to the He-ion-exposed pristine [110] surface (Fig. 5-a), pre-damaging the surface with $10 \mathrm{keV}$ $\mathrm{C}$ ions, even to higher peak $\mathrm{C}$ concentrations prior to the He ion exposures did not significantly affect the observed surface morphologies (Figs. 5-b, 5-c). This was quite surprising, since the introduced C interstitials and lattice damage resulting from the $C$ implantation was expected to greatly increase the number of near-surface trapping sites, thereby significantly reducing the incubation times thought to be required for nanofuzz formation [Meyer 2016b]. Despite varying the implanted C peak concentration by about an order of magnitude, no significant change in the SEM-measured surface pinhole size distribution was observed (Fig. 4-b).

The observation of surface ridges after He irradiation of W[110] targets has been reported over a broad energy and flux range, at $\mathrm{He}$ ion beam energies up to $30 \mathrm{keV}$ under low fluence conditions[Garrison 2015], down to He plasma impact at less than $100 \mathrm{eV}$ under high flux conditions [Kajita 2014]. Very similar ridge structures were observed at fluences similar to those of the present measurements (low to mid $10^{20} \mathrm{~cm}^{-2}$ range), but for incident fluxes more than an order of magnitude greater than those of the present investigation. For such high flux conditions, nanofuzz was eventually observed for singly crystal targets, but only at fluences significantly in excess of $10^{21} \mathrm{~cm}^{-2}$, i.e. much higher than the accumulated fluences at the flux thresholds we had observed for nanofuzz growth on polycrystalline targets [Meyer 2016b].

This suggests that the flux thresholds for nanofuzz formation on [110] single crystal W surfaces may in fact be similar to those observed for polycrystalline W surfaces [Meyer 2016b], but that the required incubation fluences for single crystal targets are significantly higher, and were in fact not reached in the present measurements. The more disordered, spaghetti-like surface morphology noted above (Fig. 3-c), was observed for the $2 \mathrm{keV} H \mathrm{He}$ ion beam exposure, where the He beam itself can cause target damage (see Table 1). However, SEM scans away from the central beam spot at this energy (Fig. 3-b) where the flux was comparable to the peak flux obtained at $218 \mathrm{eV}$, showed more ordered ridge morphologies, similar to those observed at $218 \mathrm{eV}$ (Fig. 5), suggesting that increased target damage production is not the prime reason for this morphology change. The exposures at $218 \mathrm{eV} H e$ energy of pre-damaged $\mathrm{W}$ [110] samples in comparison to those on a virgin [110] surface, which showed little change of observed surface morphology, support this conjecture. 
Several factors may contribute to longer incubation fluences for [110] single crystal W targets. First, the [110] surface is the most densely packed of all the crystallographic orientations, and therefore is characterized by significantly increased He ion reflection, requiring, for a given incident flux, a longer exposure time to build up a critical subsurface He concentration. Second, the absence of grain boundaries for the single crystal targets may inhibit He migration and trapping in the near-surface region in comparison to polycrystalline targets, as well as, more speculatively, increase the stresses required before the lattice dislocations leading to He cluster and bubble growth to occur. In that connection it is noted that MD simulations [Hammond 2014] have shown lower He retention which presumably involves deeper, larger bubbles before surface damage results for [110] surfaces in comparison to other crystallographic surface faces.

\section{Conclusions}

Surface morphologies of hot tungsten surfaces implanted using well-characterized, mono-energetic He ion beams were investigated using SEM, FIB/SEM and FIB/TEM techniques for a wide range of ion energies (218 eV to $250 \mathrm{keV}$ ), studying both polycrystalline and single-crystal tungsten targets. The formation of nanofuzz on polycrystalline tungsten under high-flux implantation of $100-k e V$ He ions enabled the determination of the flux threshold for fuzz formation at that energy, namely $0.9 \times 10^{16} \mathrm{~cm}^{-2}$

$\mathrm{s}^{-1}$, which is consistent with the energy dependence of the flux threshold found previously for lower energy implantations [Meyer 2016b]. The surface morphology of PCW implanted with 200-keV He ions did not show any nanofuzz, but did exhibit pinholing, consistent with bursting of trapped He bubbles in the near-surface region. In separate low flux high energy He-ion exposures, initial in-situ thermal desorption spectrometry measurements were performed. For the fluences investigated, He retention fractions in the range $30-50 \%$ were observed. The thermal desorption spectra showed two distinct peaks, one at $850 \mathrm{~K}$ due to He release at intrinsic $\mathrm{W}$ trapping sites, and the other at $1350 \mathrm{~K}$ due to release at damage induced trapping sites. Some saturation effects were observed in the retained He fraction as well the fluence dependence of the $1350 \mathrm{~K}$ desorption peak. Despite higher fluxes employed than those in previous measurements with PCW, nanofuzz was not observed with any of the SCW targets. Instead, pinholes and some surface roughening, ordered ridges, ridges with decreasing ridge lengths, and tangled spaghetti were observed, in order of increasing peak flux. Pre-damaging the SCW targets with $10 \mathrm{keV} \mathrm{C}$ beams to two different peak $\mathrm{C}$ concentrations did not significantly change the observed He ion induced surface morphologies. The main factor leading to the different observed surface structures appeared to be the peak He flux characterizing the different exposures. It was speculated that nanofuzz formation was not observed for any SCW target exposures because of increased incubation fluences required for such targets. Some possible reasons for such an increase were discussed. 


\section{Acknowledgements}

Research sponsored by the LDRD Program of Oak Ridge National Laboratory, managed by UT-Battelle, LLC, for the US Department of Energy, and the DOE Office of Fusion Energy Sciences. HH was appointed through the ORNL Postdoctoral Research Associates Program administered jointly by Oak Ridge Institute of Science and Education (ORISE), Oak Ridge Associated Universities (ORAU) and Oak Ridge National Laboratory (ORNL). Research supported by the Center for Nanophase Materials Sciences (CNMS), which is sponsored by the Scientific User Facilities Division, Office of Basic Energy Sciences, U.S. Department of Energy. CMP supported by Early Career Award, US Department of Energy, Office of Science, Fusion Energy Sciences. This research was performed, in part, using instrumentation (FEI Talos F200X S/TEM) provided by the Department of Energy, Office of Nuclear Energy, Fuel Cycle R\&D Program and the Nuclear Science User Facilities.

\section{References}

[Baldwin 2008] M. J. Baldwin and R. P. Doerner, "Helium induced nanoscopic morphology on tungsten under fusion relevant plasma conditions," Nucl. Fusion 48, 035001 (2008).

[Baldwin 2009] M. J. Baldwin, R. P. Doerner, D. Nishijima, K. Tokunaga, Y. Ueda, "the effects of high fluence mixed-species (deuteriuim, helium, beryllium) plasma interactions with tungsten," J. Nucl. Mat. 390-391, 886 (2009).

[Baldwin 2010] M. J. Baldwin and R. P. Doerner, "Formation of helium induced nanostructure 'fuzz' on various tungsten grades," J. Nucl. Mater. 404, 165 (2010).

[Bannister 2014] M. E. Bannister, H. Hijazi, H. M. Meyer III, V. Cianciolo, and F. W. Meyer, "Surfaceconductivity enhancement of PMMA by keV-energy metal-ion implantation," Nucl. Instrum. Methods Phys. Res. B 339, 75 (2014).

[Fu 2004] Zhang Fu, N. Yoshida, H. Iwakiri, Zengyu Xu, "Thermal desorption and surface modification of $\mathrm{He}^{+}$implanted into tungsten," J. Nucl. Mater. 329, 692 (2004).

[Garrison 2015] L. M. Garrison and G. Kulcinski, "The effects of tungsten's pre-irradiation surface condition on helium-irradiated morphology," J. Nucl. Mat. 466, 302 (2015).

[Hammond 2014] K. D. Hammond and B. D. Wirth, "Crystal orientation effects on helium ion depth distributions and adatom formation processes in plasma-facing tungsten," J. App. Phys. 116, 143301 (2014).

[Hijazi 2013] H. Hijazi and F. W. Meyer, "A large-acceptance beam-deceleration module for retrofitting into ion-source beam lines," Rev. Sci. Instrum. 84, 03330 (2013).

[Kajita 2009] S. Kajita, W. Sakaguchi, N. Ohno, N. Yoshida, and T. Saeki, "Formation process of tungsten 
nanostructure by the exposure to helium plasma under fusion relevant plasma conditions," Nucl. Fusion 49, 095005 (2009).

[Kajita 2011] S. Kajita, N. Yoshida, R. Yoshihara, N. Ohno, M. Yamagiwa, "TEM observations of the growth of helium nanobubbles on tungsten: Nanostructure formation mechanism," J. Nucl. Mat. 418, 152 (2011).

[Kajita 2014] S. Kajita, N. Yoshida, N. Ohno, Y. Hirahata, and R.Yoshihara, "Helium plasma irradiation on single crystal tungsten and undersized atom doped tungsten alloys," Phys. Scr. 89, 025602 (2014).

[Kornelsen 1970] E. V. Kornelsen, Entrapment of helium ions at (100) and (110) tungsten surfaces," Can. J. Phys. 48, 2812 (1970).

[Kornelsen 1972] E. V. Kornelsen, "The Interaction of Injected Helium with Lattice Defects in a Tungsten Crystal," Rad. Effects 13, 227 (1972).

[Kornelsen 1980] E. V. Kornelsen and A. A. Van Gorkum, "A study of He bubble nucleation in tungsten usiong thermal desorption spectrometry: cluster of 2 to 100 He atoms," J. Nucl. Mat. 92, 79 (1980).

[Krasheninnikov 2011] S. I. Krasheninnikov, "Viscoelastic model of tungsten 'fuzz' Growth," Phys. Script. T145, 014040 (2011).

[Lasa 2014] A. Lasa, K. Tähtinen, and K. Nordlund, "Loop-punching and bubble rupture causing surface roughening - A model for W fuzz growth," Eur. Phys. Lett. 105, 25002 (2014).

[Lee 2007] H. T. Lee, A. A. Haasz, J. W. Davis, R. G. Macaulay-Newcombe,"Hydrogen and helium trapping in tungsten under single and sequential irradiations," J. Nucl. Mat. 360, 196 (2007).

[Meyer 2000] F.W. Meyer, "ECR-based Atomic Collision Physics Research at ORNL MIRF", in Trapping Highly Charged lons: Fundamentals and Applications, J. Gillaspy, ed., (Nova Science Pub., New York, 2000), pp. 117-164.

[Meyer 2006] F. W. Meyer et al., "The ORNL Multicharged Ion Research Facility Upgrade project," Nucl. Instrum. Methods Phys. Res. B 242, 71 (2006).

[Meyer 2014] F. W. Meyer et al., "He-ion and self-atom induced damage and surface-morphology changes of a hot W target," Phys. Scr. T159, 014209 (2014).

[Meyer 2016a] F. W. Meyer, L. Han, H. Hijazi, M. E. Bannister, K. A. Unocic, C. M. Parish, P. S. Krstic, Abstract, $22^{\text {nd }}$ PSI, Rome, Italy, 2016.

[Meyer 2016b] F. W. Meyer et al., "Flux threshold measurements of He-ion beam induced nanofuzz formation on hot tungsten surfaces," Phys. Scr. T167, 014019 (2016). 
[Miyamoto 2014] M. Miyamoto, T. Watanabe, H. Nagashima, D. Naishijima, R. P. Doerner, S. I. Krasheninnikov, A. Sagara, and N. Yoshida, "In situ transmission electron microscope observation of the formation of fuzzy structures on tungsten," Phys. Scr. T159, 014028 (2014).

[Ohno 2013] N. Ohno et al., "Influence of crystal orientation on damages of tungsten exposed to helium plasma," J. Nucl. Mat. 438, S879 (2013).

[Parish 2013] C. M. Parish, H. Hijazi H, H. M. Meyer, and F. W. Meyer, "Effect of tungsten crystallographic orientation on He-ion-induced surface morphology changes," Acta Mater. 62, 173 (2013).

[Sefta 2013] F. Sefta, K. D. Hammond, N. Juslin, and B. D. Wirth, "Tungsten surface evolution by helium bubble nucleation, growth, and rupture," Nuc. Fusion 53, 073015 (2013).

[Stoller 2013] R. E. Stoller, M. B Toloczko, G. S. Was, A. G. Certain, S. Dwaraknath, and F. A. Garner, "On the use of SRIM for computing radiation damage exposure," Nuc. Instrum. Methods Phys. Res. B. 310, 75 (2013).

[Takamura 2006] S. Takamura, N. Ohno, D. Nishijima, and S. Kajita, "Formation of Nanostructured Tungsten with Arborescent Shape due to Helium Plasma Irradiation," Plasma Fusion Res. 1, 051 (2006).

[Wright 2015] G. M. Wright, G. G. van Eden, L. A. Kesler, G. De Temmerman, D. G. Whyte, and K. B. Woller, "Characterizing the recovery of a solid surface after tungsten nano-tendril formation," J. Nucl. Mater. 463, 294 (2015).

[Ziegler 1985] TRIM (Transport and Range of lons in Matter), The Stopping and Range of Ions in Solids, J. F. Ziegler, et al., eds. (New York: Pergamon, 1985). 
Table 1. [110] Single crystal exposure conditions. The $C$ beam implant energy for exposures 2 and 3 was $10 \mathrm{keV}$; the average damage dose was estimated from the SRIM result for the number of target vacancies produced per incident ion, and the maximum penetration depth, as described in [Meyer 2016b], using the peak He or $\mathrm{C}$ fluences of the exposure. Target temperature was $1000^{\circ} \mathrm{C}$.

\begin{tabular}{|l|l|l|l|l|l|}
\hline $\begin{array}{l}\text { Exposure } \\
\text { number }\end{array}$ & $\begin{array}{l}\mathrm{He} \\
\text { Energy }\end{array}$ & $\begin{array}{l}\text { Peak He } \\
\text { flux } \\
\left(\mathrm{He} / \mathrm{cm}^{2} \mathrm{~s}\right)\end{array}$ & $\begin{array}{l}\text { Peak He } \\
\text { fluence } \\
\left(\mathrm{He} / \mathrm{cm}^{2}\right)\end{array}$ & $\begin{array}{l}\text { Peak C } \\
\text { density } \\
\left(\mathrm{C} / \mathrm{cm}^{3}\right)\end{array}$ & $\begin{array}{l}\text { Average } \\
\text { damage } \\
\text { dose } \\
(\mathrm{dpa})\end{array}$ \\
\hline 1 & $218 \mathrm{eV}$ & $5.4 \times 10^{16}$ & $2.2 \times 10^{20}$ & 0 & 0 \\
\hline 2 & $218 \mathrm{eV}$ & $5.0 \times 10^{16}$ & $1.8 \times 10^{20}$ & $8.4 \times 10^{22}$ & 8.6 \\
\hline 3 & $218 \mathrm{eV}$ & $5.4 \times 10^{16}$ & $1.9 \times 10^{20}$ & $8.4 \times 10^{23}$ & 86 \\
\hline 4 & $2 \mathrm{keV}$ & $1.0 \times 10^{17}$ & $3.7 \times 10^{20}$ & 0 & 228 \\
\hline
\end{tabular}


(a)
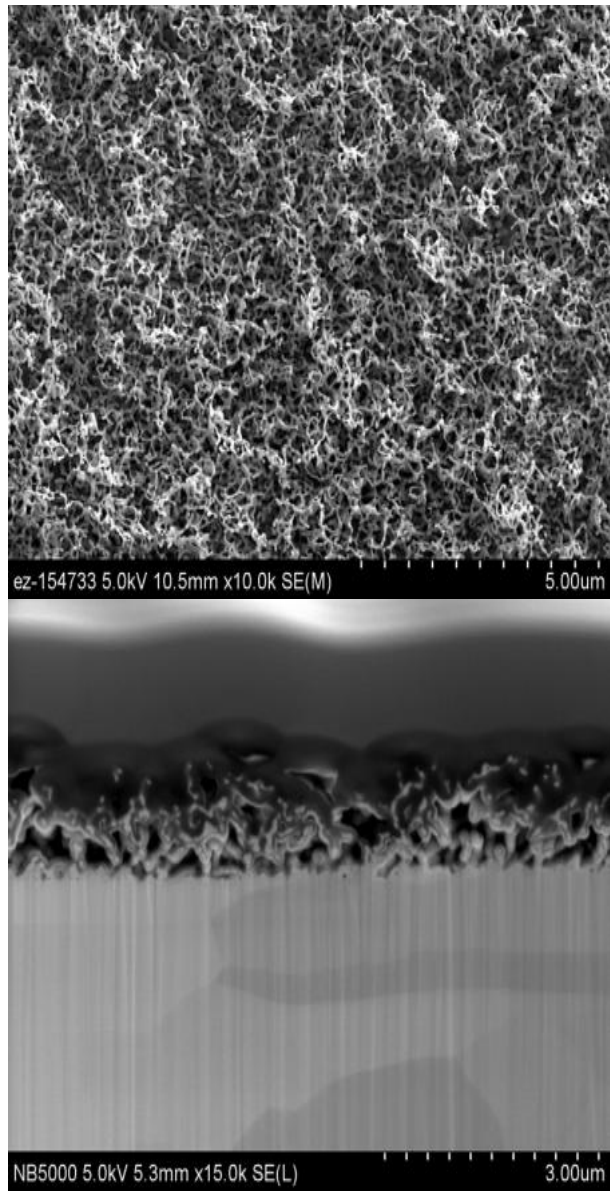

(d) (b)

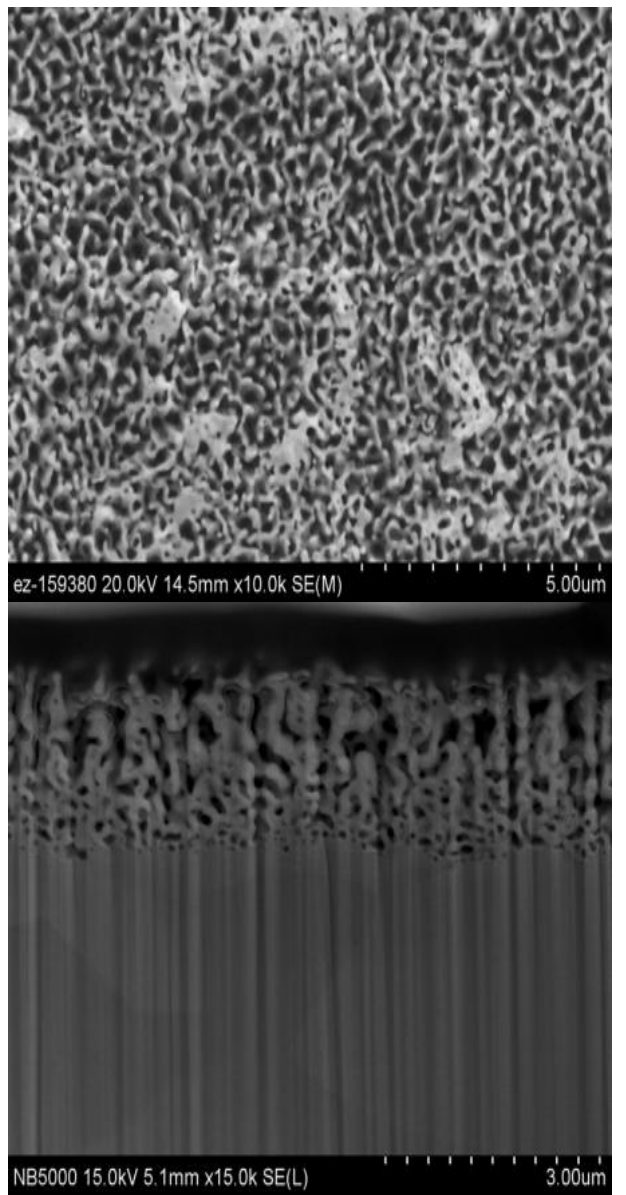

(c)

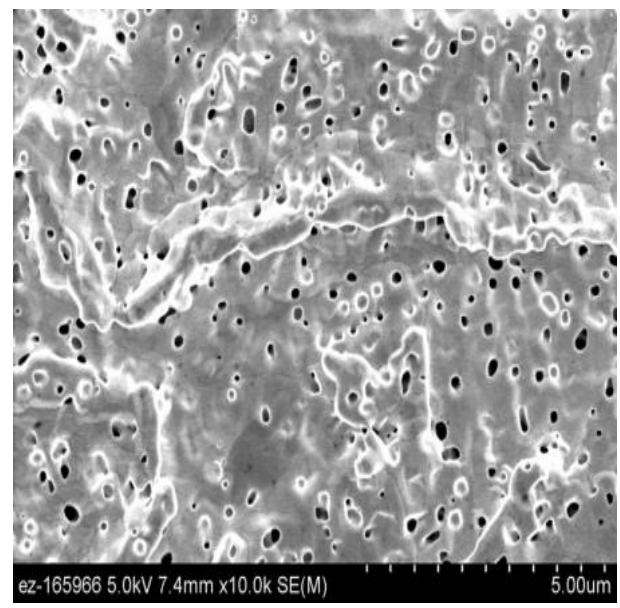

(e)

Figure 1.

SEM images of polycrystalline tungsten after implantation with He ions: (a) energy $218 \mathrm{eV}$, flux $3.1 \mathrm{x}$ $10^{16} \mathrm{~cm}^{-2} \mathrm{~s}^{-1}$, fluence $2.2 \times 10^{20} \mathrm{~cm}^{-2}, \mathrm{~W}$ temperature $1300 \mathrm{~K}$; (b) energy $100 \mathrm{keV}$, flux $1.9 \times 10^{16} \mathrm{~cm}^{-2} \mathrm{~s}^{-1}$, fluence $1.0 \times 10^{19} \mathrm{~cm}^{-2}$, W temperature $1200 \mathrm{~K}$; (c) energy $200 \mathrm{keV}$, flux $1.1 \times 10^{16} \mathrm{~cm}^{-2} \mathrm{~s}^{-1}$, fluence $1.0 \times$ $10^{19} \mathrm{~cm}^{-2}, \mathrm{~W}$ temperature $1250 \mathrm{~K}$. FIB/SEM images of cross section of near surface region of PCW after He-ion implantation: (d) energy $218 \mathrm{eV}$; (e) energy $100 \mathrm{keV}$. No FIB/SEM imaging was performed on the 200-keV exposed target since no nanofuzz was observed in the SEM image. 


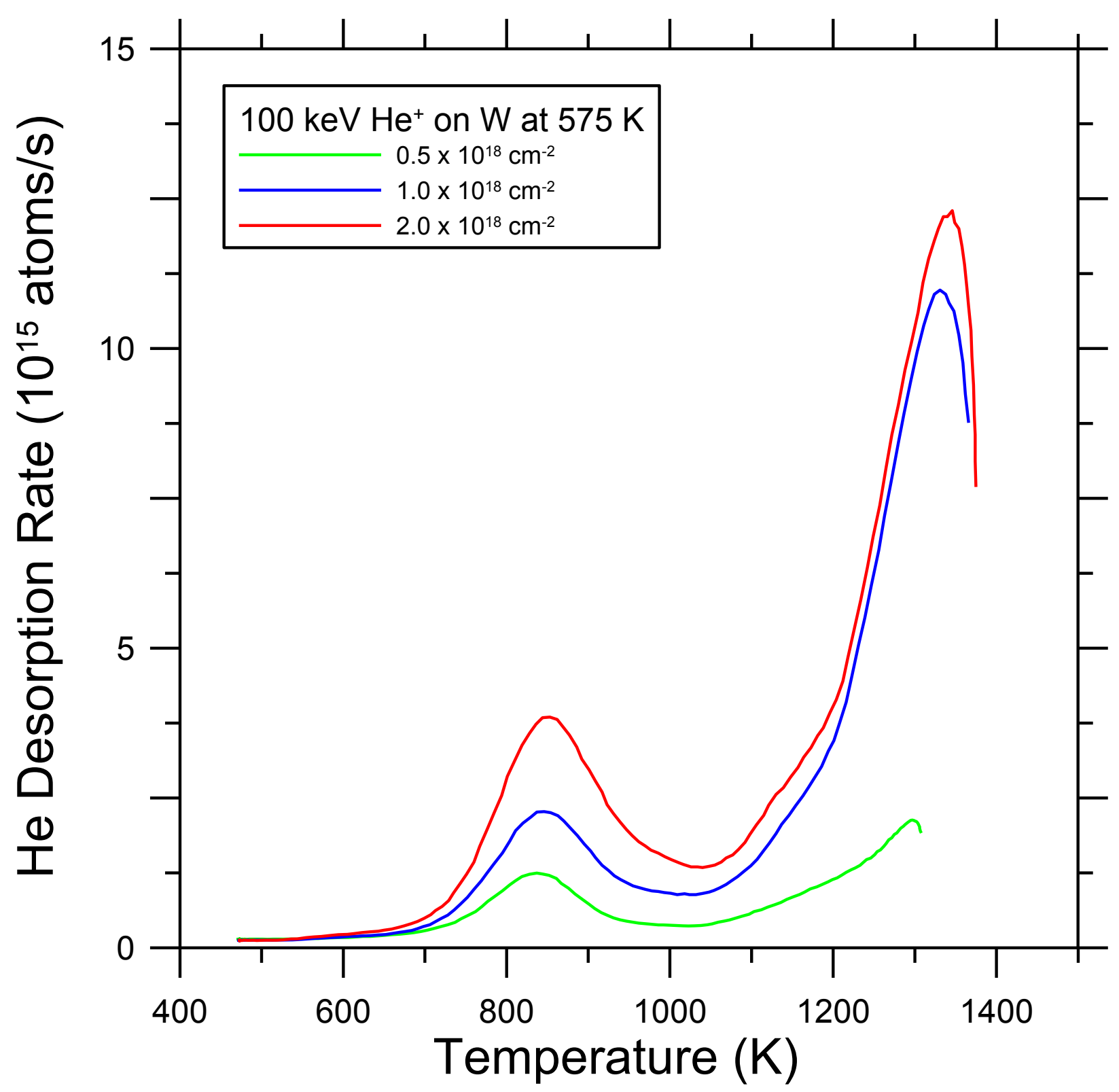

Figure 2.

Thermal desorption spectra of polycrystalline tungsten after sequential implantations with $100 \mathrm{keV}$ He ions (flux $4.3 \times 10^{14} \mathrm{~cm}^{-2} \mathrm{~s}^{-1}$ ) at a sample temperature of $575 \mathrm{~K}$. 


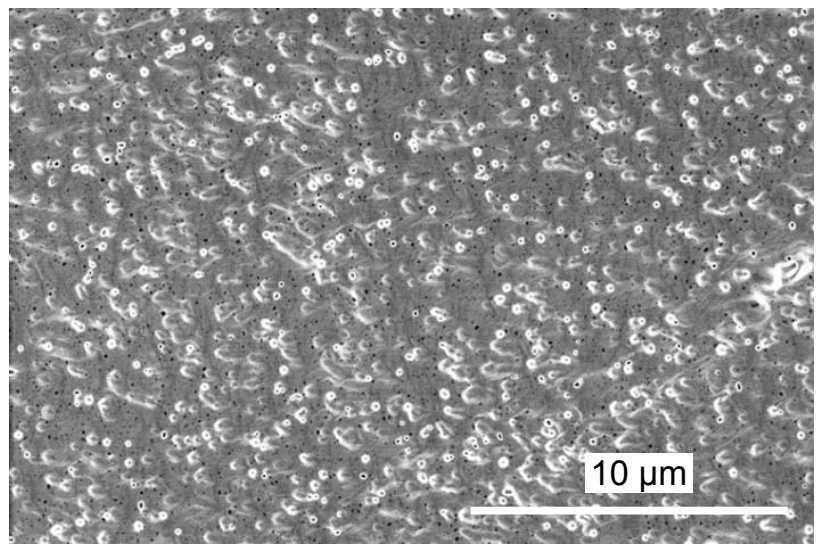

(a)

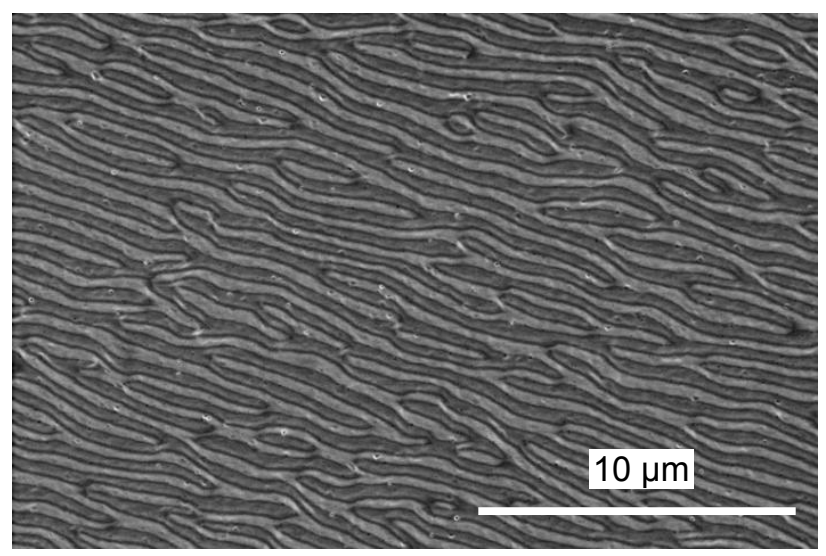

(b)

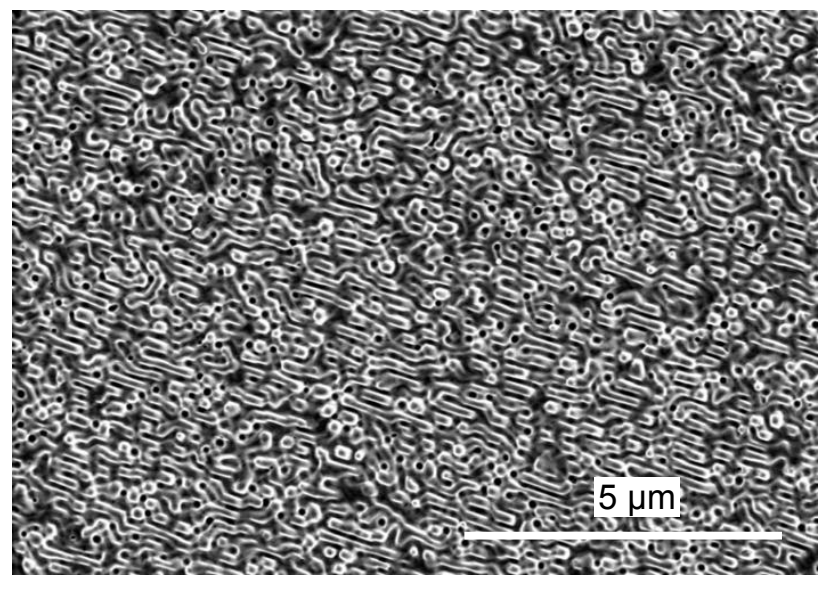

(c)

Figure 3.

SEM images showing evolution of observed surface morphologies on He-implanted single-crystal [110] tungsten with increasing ion flux: (a) roughening and pinholes, (b) ordered ridges, to (c) tangled spaghetti. All images from exposure \#4. See text and Table 1 for details of exposures. 


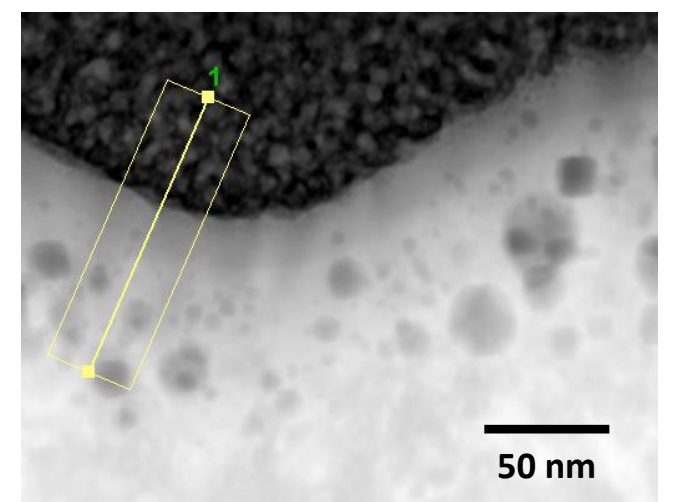

(a)

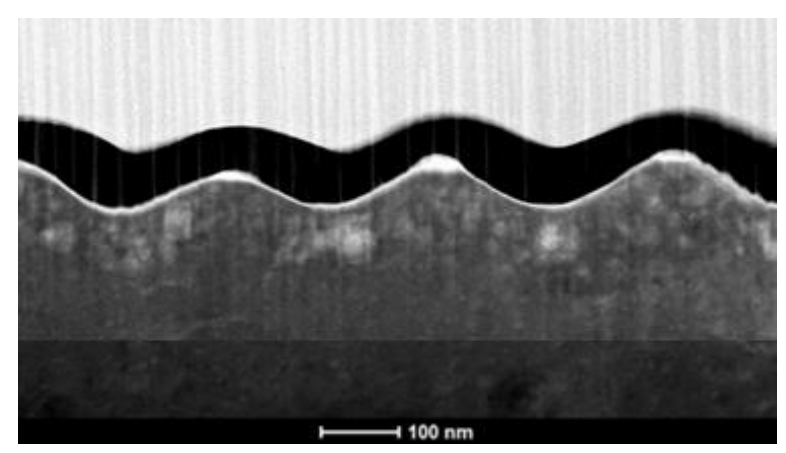

(c)

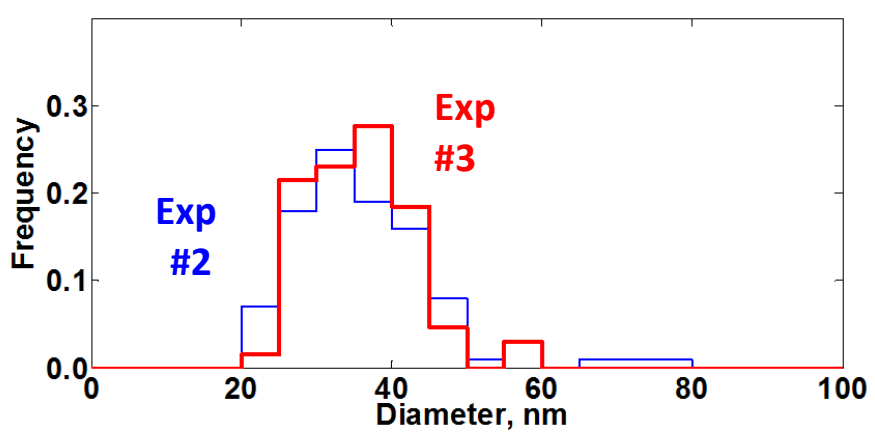

(b)

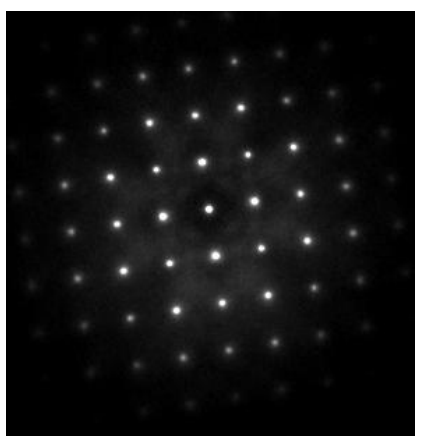

(d)

Figure 4.

FIB-TEM imaging of an He-exposed W[110] surface pre-damaged by $10 \mathrm{keV} C$ ion exposure (Exposure \#3 in Table 1): (a) He bubbles in the near surface region (the yellow rectangle indicates region where elemental analysis was done, which is not shown), (b) comparison of the SEM-measured surface pinhole size distribution for exposures \#2 and \#3 (Table 1), (c) FIB cut transverse to ridge line showing ordered ripple structure, (d) electron diffraction pattern of FIB slice, showing $\langle 100\rangle$ alignment of ridge line. 


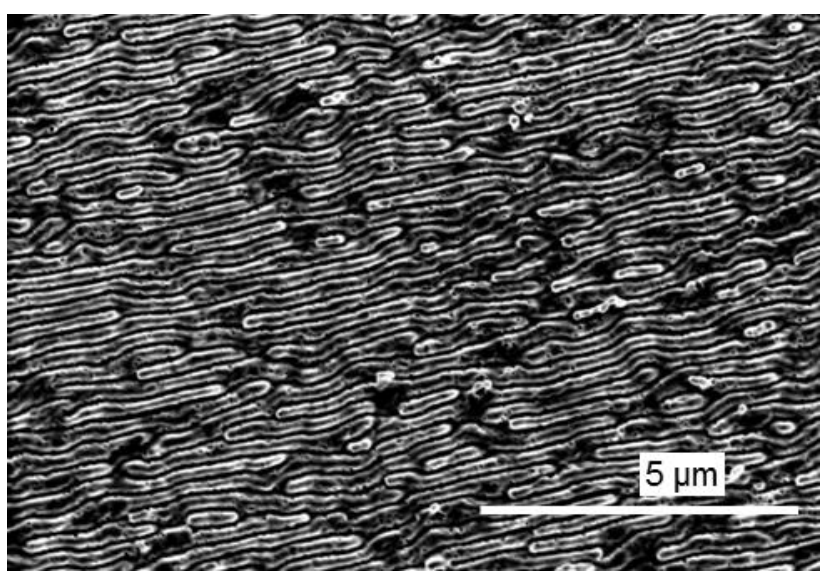

(a)

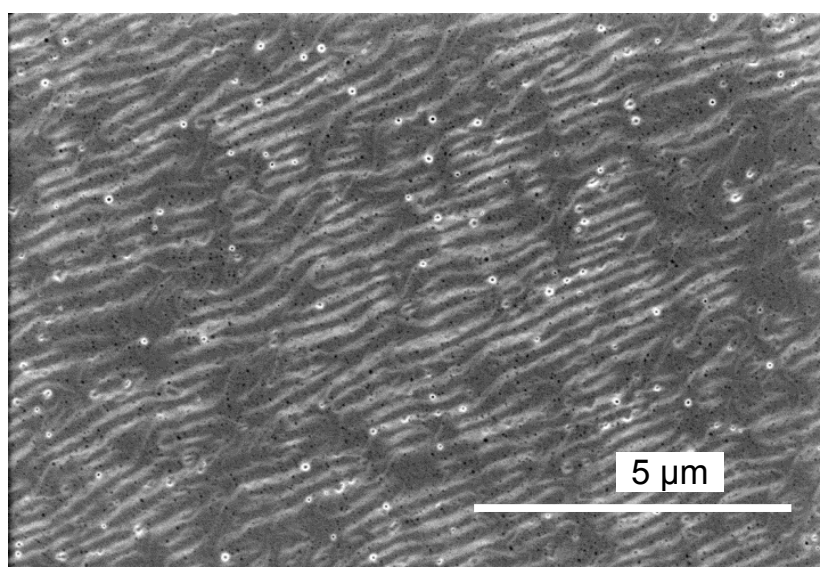

(b)

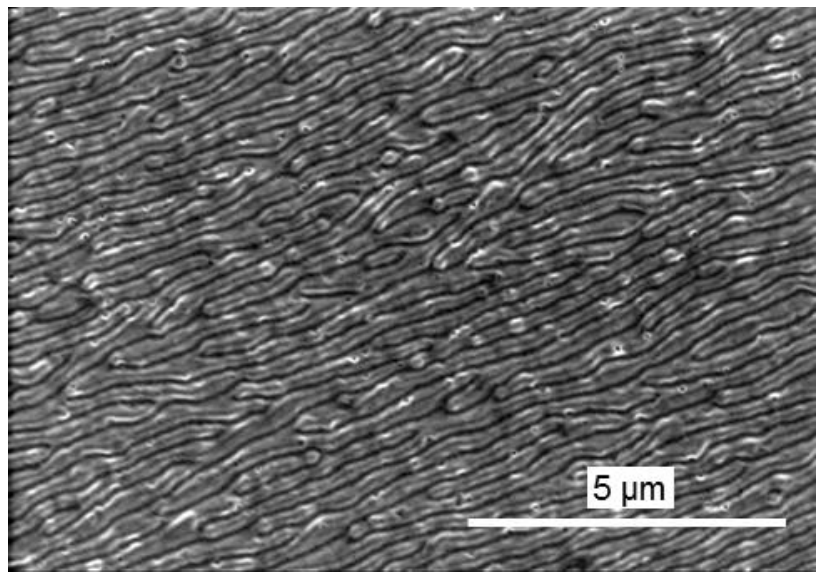

(c)

Figure 5.

SEM images of observed surface morphologies on He-implanted single-crystal [110] tungsten with increasing $C$ impurities: (a) pristine surface (exposure $\# 1$ of Table 1 ), (b) C impurities at peak density of $8.4 \times 10^{22} \mathrm{~cm}^{-3}$ (exposure \#2), (c) C impurities at peak density of $8.4 \times 10^{23} \mathrm{~cm}^{-3}$ (exposure \#3). See text and Table 1 for details of exposures. 